Revista del Centro de Investigación de la Universidad La Salle

Vol. 14, No. 55, Enero-Junio, 2021: 103-126

DOI: http://doi.org/10.26457/recein.v14i55.2794

\title{
Influencia entre el Número de Colaboradores y Estrategias en las MIPyMEs de la Zona Centro del Estado de Veracruz
}

\section{Influence between the Number of Collaborators and Strategies in the MSMEs of the Downtown Area of the State of Veracruz}

\author{
Claudia Vásquez Rojas* \\ Universidad Popular Autónoma del Estado de Puebla (México) \\ Daniel Martínez Navarrete \\ Universidad Tecnológica del Centro de Veracruz (México) \\ Alejandro de Jesús Hernández Peralta \\ Universidad Tecnológica del Centro de Veracruz (México) \\ Daniel Villanueva Vásquez \\ Centro de Investigación e Innovación en Tecnologías de la Información \\ y Comunicación (México)
}

Recibido: 06 de octubre de 2020 Aceptado: 03 de diciembre de 2020

Publicado: 31 de mayo de 2021

\section{Resumen}

Actualmente, el sector empresarial es uno de los principales factores que impulsan y fortalecen el desarrollo de un país, en ese sentido, las MIPyMEs juegan un papel fundamental, sin embargo, a pesar de su impacto positivo se ven en la necesidad de afrontar

*Email: claudia.vasquez@live.com.mx

Revista del Centro de Investigación. Universidad La Salle por Dirección de Investigación. Universidad La Salle Ciudad de México se distribuye bajo una Licencia Creative Commons Atribución-NoComercial-Compartirlgual 
Vásquez Rojas, C.; Martínez Navarrete, D.; Hernández Peralta, A. J.; Villanueva Vásquez, D.

los cambios globales y sobrevivir en un mercado altamente competitivo. El plan estratégico es un proceso imperativo para guiar a una organización en el logro de sus objetivos, las MIPyMEs cuentan con recursos limitados que deben ser eficazmente coordinados para que la empresa pueda ser competitiva, dentro de este contexto, conocer la influencia entre las estrategias y la fuerza laboral, coadyuva en la decisión de establecer un número determinado de estrategias de acuerdo con el número de colaboradores. Se empleó un modelo de regresión de análisis de covarianza (ANCOVA) para estudiar la dependencia entre las variables en dos periodos dados; se consideraron cuarenta y un casos de éxito de diferentes empresas identificando el número de colaboradores y las estrategias que han implementado. Se realizó una correlación lineal de Pearson entre las variables número de colaboradores y número de estrategias, donde, se presentó una correlación fuerte y positiva del $71.16 \%$ para la totalidad de un periodo de dos años, se observó que la relación entre dichas variables fue alta. Los resultados del modelo econométrico ANCOVA sugieren que, de acuerdo con el valor estimado del parámetro $\beta_{2}$ por cada aumento en el número de colaboradores correspondiente a una unidad, se espera que en promedio el número de estrategias de las MIPyMEs aumente en 0.3291, este efecto incremental es independiente del periodo en el que se observe y al ser positivo confirma la dependencia positiva de las estrategias en función del número de colaboradores. Los resultados de la propuesta permiten estimar el número de estrategias que se recomienda implementar en función del número de colaboradores que la integran, lo anterior es fundamental para administrar los recursos que se requieren.

Palabras clave: Estrategias, Recursos humanos, MIPyMEs, ANCOVA. 


\section{Abstract}

Nowadays, the business sector is one of the main factors that promote and strengthen the development of a country, in that sense, MSMEs play a fundamental role, however, despite their positive impact, they have the need to face global changes and survive in a highly competitive market. The strategic plan is an imperative process to guide an organization in achieving its objectives, MSMEs have limited resources that must be effectively coordinated so that the company can be competitive, within this context, know the influence between strategies and the workforce contributes in the decision to establish a certain number of strategies according to the number of collaborators. An analysis of covariance regression model (ANCOVA) was carried out to study the dependence between the variables in two periods; forty-one case studies from different companies were considered, identifying the number of collaborators and the strategies they have implemented. A linear Pearson correlation was performed between the variables number of collaborators and number of strategies, where a strong and positive correlation of $71.16 \%$ was presented for the entire period, it is reflected that the relationship between said variables was high. The results of the ANCOVA econometric model suggest that, according to the estimated value of the parameter $\beta_{2}$ for each increase in the number of collaborators corresponding to a unit, it is expected that on average the number of strategies of the MSMES will increase by 0.3291 , this incremental effect is independent of the period in which it is observed and, since it is positive, it confirms the positive dependence of the strategies based on the number of collaborators. The results of the proposal allow estimating the number of strategies that is recommended to implement based on the number of collaborators that integrate it, the above is essential to manage the resources that are required.

Keywords: Strategies, Human Resources, MSEs, ANCOVA 
Vásquez Rojas, C.; Martínez Navarrete, D.; Hernández Peralta, A. J.; Villanueva Vásquez, D.

\section{Introducción}

En los países en vías de desarrollo y de economía emergente de cualquier parte del mundo es fundamental la identificación de las principales variables que afectan el crecimiento de la economía local y nacional (Vrgovic et al., 2012), y, de entre las múltiples variables que se han publicado en la literatura, el desarrollo y fortalecimiento de las Micro, Pequeñas y Medianas Empresas (MIPyMEs) es considerado como una variable que ejerce efectos positivos en el desarrollo de una nación (Jaffe \& Lerner, 2006).

México no es la excepción, de acuerdo con Antonio et al., (2020) se ha identificado que, el 99.8\% de las unidades económicas son MIPyMEs, las cuales aportan el 52.2\% del PIB y generan el 69.4\% de empleo del país. Pérez-Elizundia et al., (2020) argumentan que, las MIPyMEs convergen con otros países respecto a su aportación en la economía nacional, haciendo especial énfasis en que las empresas mexicanas tienen en promedio diez o menos colaboradores. Este aspecto, las posiciona con una menor capacidad para afrontar y mejorar sus procesos empresariales.

A pesar del impacto en el desarrollo económico, las MIPyMEs se enfrentan a diferentes limitaciones de recursos, financiamiento, infraestructura y gestión (Amarjit \& Nahum, 2012; Molina Sánchez et al., 2014; Obando Changúan et al., 2020; Zaridis \& Mousiolis, 2014) disponiendo de menos herramientas para mejorar el desempeño respecto a las grandes empresas. Sin embargo, un recurso común en todas las organizaciones, que ha sido objeto de una creciente atención teórica, empírica y práctica es el de los recursos humanos (Sheehan, 2013).

Ante el acelerado crecimiento global, las organizaciones buscan una continua armonía entre sus clientes, colaboradores, proveedores entre otros involucrados (Aguirre, 2015; Ahumada Tello \& Perusquia Velasco, 2016). Algunos estudios sugieren (Ait Razouk, 2011; Patel \& Cardon, 2010) que, la adopción de prácticas específicas de gestión de recursos humanos puede contribuir a mejorar el desempeño de las MIPyMEs y mantener la competitividad.

En ese sentido, los colaboradores juegan un rol importante en cualquier organización y más aún en el caso de las MIPyMEs, debido a que, dentro de las múltiples actividades que 
desempeñan, la participación en el proceso de planeación estratégica es fundamental para el desempeño de esta.

Existe un vasto campo en los temas de estrategias empresariales y su integración con las acciones para mejorar la competitividad, haciendo crecer y crear riqueza (Hitt et al., 2008; Ireland et al., 2001), si bien, tales temas, están relacionados con las capacidades y recursos (Wang \& Ang, 2004), señalando que las MIPyMEs cuentan con pocos recursos (Knight, 2001), pero esta debilidad es compensada con la flexibilidad y adaptabilidad debido a la simplicidad de su estructura, técnicas y procesos, ante los cambios del entorno (AragónSánchez \& Sánchez-Marín, 2005; Messeghem, 2003; Narula, 2004; Ong et al., 2010; Pil \& Holweg, 2003).

Con respecto a los modelos econométricos en el área de planeación estratégica resultan importantes para la toma de decisiones, como el estudio que realizaron Islami et al. (2020), donde analizan tres estrategias genéricas del modelo de Porter (estrategia bajo costo, estrategia diferenciación y estrategia de enfoque) y su relación con el desempeño de 113 empresas pequeñas y medianas que operaban bajo entornos competitivos, mediante la aplicación de un instrumento y la utilización de un modelo econométrico para medir las relaciones. No obstante, solo se enfocan a analizar tres estrategias genéricas de las cinco a las que se guía el modelo de las cinco fuerzas de Porter, asimismo, el articulo menciona que algunos estrategas pueden implementar estrategias incorrectas, omitiendo que a largo plazo pueden devastar a su organización.

Por otro lado, el análisis ANCOVA ha sido utilizado en diversos campos de la ciencia como uno de los principales métodos de análisis estadísticos, dejando entre ver, que es una herramienta eficaz en las investigaciones científicas aplicadas (Shieh, 2020). Los autores presentan un estudio donde investigaron el impacto de la estrategia corporativa y sus puntajes de reputación analizando los diferentes tipos de estrategias y algunos factores que influyen en la reputación, como, el tamaño de la empresa, desempeño, relación deuda-capital, tipo de propiedad, tipos de actividad, exposición de medios, utilizando el modelo ANCOVA el cual demostró una correlación positiva entre los factores (Williams et al., 2005).

Lussier y Sonfield (2012), realizaron un estudio acerca de las empresas familiares en siete países donde examinaron las covariables de antigüedad, número de empleados, industria y tipo de propiedad, para probar que existen diferencias en la planeación de la sucesión de 
Vásquez Rojas, C.; Martínez Navarrete, D.; Hernández Peralta, A. J.; Villanueva Vásquez, D.

empresas familiares, a través del análisis ANCOVA, lo cual permitió identificar aquellos países que tenían contrastes significativos. Sin embargo, este estudio está enfocado únicamente a empresas familiares y a realizar una comparación entre otros países, aunque, se analizó el número de colaboradores como una covariable, es importante destacar que el artículo la consideró dentro de un proceso de planeación de sucesión.

Actualmente, las empresas están perdiendo la sinergia para encontrar métodos que les permitan mantener una posición en el mercado. Por lo tanto, es significativo proponer o mejorar procesos que sirvan para alcanzar sus metas. Existen varios estudios enfocados a los factores competitivos en las MIPyMEs que muestran que la estructura y administración de los recursos humanos contribuyen a la competitividad y consecuentemente al éxito (Fiegenbaum \& Karnani, 1991). Barney (1991) resalta la valiosa contribución de los recursos intangibles de una organización para crear ventaja competitiva sostenible. Actualmente las estrategias juegan un rol sustancial en la gestión del desempeño empresarial para alcanzar los objetivos planteados por las MIPyMEs mediante el uso de sus recursos humanos (Leal de Valor et al., 2011).

La gestión de recursos humanos en pequeñas empresas puede ser más eficaz al momento de generar convencimiento e involucramiento de los colaboradores al existir menos jerarquización (Kuratko et al., 2001). Por otro lado, si no hay colaboradores, nadie realizaría acciones organizacionales, por ende, la integración de los recursos humanos en el desarrollo de un plan estratégico, debe ser considerado como el primer paso (Management, 2011; Rocca et al., 2016).

La evolución de los diferentes procesos de gestión de recursos humanos, ha dado un giro a los conceptos que se han manejado durante décadas como la consideración del término "empleados" que se traducía como un gasto necesario a cambio de la realización de algún "trabajo", actualmente, el término ha ido evolucionando en el ámbito organizacional hasta llegar al concepto de "colaboradores" los cuales son piezas fundamentales para el establecimiento de estrategias, el logro de objetivos y metas (Gibert, 2006). Los colaboradores tienen la visión de agregar valor a la organización a través de sus habilidades, aptitudes y actitudes afines a las actividades para generación de productividad (López Fernández et al., 2018). De aquí que, las organizaciones tienen una nueva ideología de 
incorporar, capacitar y buscar el crecimiento y desarrollo de los colaboradores mediante la motivación (Santini et al., 2017).

La mayoría de las organizaciones llevan a cabo un análisis de las competencias (Dullayaphut \& Untachai, 2013; Muda \& Rahman, 2016) profesionales de cada uno de sus integrantes para que puedan ser capaces de tomar las decisiones y resolver los problemas que se les presentan en las áreas que colaboran. Un paso importante en la previsión de colaboradores es el establecimiento de una misión, las funciones y describir todas las actividades a realizar en el puesto y a partir de ellas se definen las evaluaciones de desempeño a través del cumplimiento de objetivos e indicadores (Palombino Primo et al., 2014). Por lo tanto, resulta importante establecer en las MIPyMEs un número adecuado de estrategias de acuerdo con el número de colaboradores, definiendo de esta manera la capacidad y la calidad de la fuerza laboral con la que cuenta una empresa para poder llevar a cabo el plan estratégico. A partir de ello, se constituyen las siguientes hipótesis:

$\mathrm{H}_{0}$ : No existe relación entre las variables número de estrategias empresariales y número de colaboradores.

$\mathrm{H}_{\mathrm{a}}$ : Eiste relación entre las variables número de estrategias empresariales y número de colaboradores.

El presente trabajo tiene como principal objetivo identificar si existe una relación estadísticamente significativa entre los integrantes de una organización y la determinación de un número adecuado de estrategias empresariales que se deben llevar a cabo para ser competitiva y asegurar su permanencia en el mercado.

\section{Materiales y Métodos}

La planeación estratégica es un proceso analítico del entorno que rodea a una organización, que conlleva a la identificación de la situación actual para el establecimiento de estrategias, metas y objetivos para alcanzar la competitividad (Estrada \& Heijs, 2018), es decir, la planeación estratégica brinda las pautas para el establecimiento de acciones que conduzcan con el desarrollo óptimo de una organización (Jaramillo, 2019). Las MIPyMEs poseen una 
Vásquez Rojas, C.; Martínez Navarrete, D.; Hernández Peralta, A. J.; Villanueva Vásquez, D.

flexibilidad en su estructura que da pie a una rápida adaptabilidad de todos sus recursos para enfrentar los cambios del entorno (Messeghem, 2003).

Se obtuvo una muestra representativa de las MIPyMEs establecidas en empresas de la zona centro del estado de Veracruz considerando el Sistema de Clasificación Industrial de América del Norte (SCIAN-2018) y el Directorio Estadístico Nacional de Unidades Económicas (DENUE 2018), analizando únicamente la actividad económica de restaurantes con servicio de preparación de alimentos a la carta, con una población de 341 empresas. De las cuales no todas contaban con un plan estratégico, por lo tanto, se aplicó un muestreo por cuotas en el que se recopiló información de las empresas donde se ha aplicado un modelo de planeación estratégica; para efecto del presente trabajo se elaboró una base de datos de MIPyMEs de 41 empresas que han llevado a cabo el diseño de estrategias en sus planes estratégicos en un período comprendido para los años 2017 y 2018 (Tabla 1).

\section{Tabla 1}

Distribución de la Muestra

\begin{tabular}{lcccc}
\hline Sector & Total & $\begin{array}{c}\text { Micro (0-10 } \\
\text { integrantes) }\end{array}$ & $\begin{array}{c}\text { Pequeña (11-50 } \\
\text { integrantes) }\end{array}$ & $\begin{array}{c}\text { Mediana (51-100 } \\
\text { integrantes) }\end{array}$ \\
\hline Servicios & 341 & 297 & 41 & 3
\end{tabular}

Fuente: Elaboración propia con datos de DENUE y SCIAN

El muestreo por cuotas puede considerarse un muestreo por juicio de dos etapas: desarrollando categorías de control con base en características relevantes de la población y, estableciendo las proporciones de los elementos de la muestra o de cada cuota que poseen las categorías de control (Malhotra, 2012; Ochoa, 2015), de tal forma que sean iguales a la proporción de los elementos de la población.

Las empresas que diseñaron estrategias y el tamaño de la empresa, fueron las categorías de control para calcular las cuotas, a partir de estas variables se aplicó un muestreo no probabilístico por conveniencia permitiendo seleccionar las MIPyMEs que, por sus implicaciones solo pudieron ser aceptadas en el estudio (Otzen \& Manterola, 2017) al 
encontrarse dentro de las pocas que establecieron estrategias, por lo anterior, se determinó un tamaño de muestra de 41 unidades económicas.

Siguiendo el procedimiento de Lohr (2015), se calculó el peso proporcional $w_{i}$ de cada cuota con respecto al total de la población y, posteriormente se multiplicó por el tamaño de la muestra, dando lugar al número de empresas para cada cuota como se describe en la Tabla 2, las empresas medianas fueron excluidas de las cuotas debido a que el valor de 0.36 es muy cercano a 0 , por lo tanto no fue significativo.

\section{Tabla 2}

Tamaño de las Cuotas que Conforman la Muestra

\begin{tabular}{|c|c|c|c|}
\hline Cuota & $\begin{array}{c}\text { Total de } \\
\text { establecimientos } \\
\boldsymbol{X}_{\boldsymbol{i}}\end{array}$ & $\begin{array}{c}\text { Pesos } \\
\boldsymbol{w}_{\boldsymbol{i}}=\frac{\boldsymbol{X}_{\boldsymbol{i}}}{\sum_{\boldsymbol{i}=\mathbf{1}}^{\mathbf{2}} \boldsymbol{X}_{\boldsymbol{i}}}\end{array}$ & Cuotas precisas \\
\hline Microempresas & 297 & $w_{i}=\frac{297}{341}=0.87$ & $C_{1}=0.87 * 41 \approx 36$ \\
\hline $\begin{array}{l}\text { Pequeñas } \\
\text { empresas }\end{array}$ & 41 & $w_{i}=\frac{41}{341}=0.12$ & $C_{2}=0.12 * 41 \approx 5$ \\
\hline Medianas & 3 & $w_{i}=\frac{3}{341}=0.01$ & $C_{3}=0.12 * 41 \approx 0$ \\
\hline Total & 341 & Total & 41 \\
\hline
\end{tabular}

Fuente: elaboración propia con datos del DENUE y SCIAN

Se empleó un modelo de regresión de análisis de covarianza (ANCOVA) el cual permite controlar el efecto parcial de las variables independientes de distintos grupos (Huitema, 2011; Rheinheimer \& Penfield, 2001; Valente \& MacKinnon, 2017; Van Breukelen, 2013) que conforman la muestra sobre la variable dependiente a través de variables dicótomas (Gujarati \& Porter, 2011). El modelo ANCOVA permite observar las diferencias en el valor estimado promedio de la variable dependiente entre un determinado número de categorías mutuamente excluyentes en las cuales se divide la muestra y que definen el uso de variables dicótomas, éstas se denotan por $D_{t}$ cuando se trata de distintos periodos y $D_{i}$ cuando se trata de distintos grupos u objetos. Al ser variables dicótomas asumen sólo dos valores: 1 para denotar la 
Vásquez Rojas, C.; Martínez Navarrete, D.; Hernández Peralta, A. J.; Villanueva Vásquez, D.

presencia de la clasificación de la muestra que se está observando y 0 para denotar su ausencia.

Para el diseño de los modelos ANCOVA, es conveniente elegir una categoría base o de comparación con las demás categorías de la muestra, para lo cual el parámetro estimado de intersección $\beta_{0}$ estimará el valor promedio de la variable dependiente para dicha categoría, mientras que cada parámetro estimado del modelo para cada variable dicótoma $\beta_{1}, \beta_{2}, \ldots, \beta_{k}$ representará la diferencia en el valor promedio de la variable dependiente correspondiente a la categoría que asume el valor de 1 en relación a la categoría base, de ahí que estos puedan identificarse como coeficientes de intercepto diferencial, la regla general para introducir un número adecuado de variables dicótomas es restar 1 al número total de categorías o clasificaciones de la muestra para evitar un problema de colinealidad perfecta (Wooldridge, 2001).

De manera general la Ecuación 1 muestra un modelo ANCOVA que permite controlar el efecto incremental de una variable cuantitativa sobre el valor promedio estimado de la variable dependiente a través de una variable dicótoma que clasifica la muestra en dos periodos de observación independientes.

$$
Y_{t}=\beta_{0}+\beta_{1} D_{1 t}+\beta_{2} X_{t}+u_{t}
$$

Si $D_{1 t}=0$, entonces el valor promedio estimado de la variable dependiente para el primer periodo correspondiente a la categoría base de la muestra es $\beta_{0}$, en caso contrario, si $D_{1 t}=$ 1 , entonces el valor promedio estimado para el segundo periodo es $\beta_{0}+\beta_{1}$, de ahí que $\beta_{1}$ puede ser un parámetro de intercepto diferencial ya que nos da una medida de la diferencia entre el valor promedio de la variable dependiente que asume en el segundo periodo en comparación con el primero. Por último, el parámetro $\beta_{2}$ explica el incremento en el valor promedio estimado de la variable dependiente cuando $X_{t}$ aumenta en una unidad, independientemente del periodo en el que se esté observando.

El modelo econométrico ANCOVA para estudiar la dependencia del número de estrategias de acuerdo con el número de colaboradores de las MIPyMEs en dos periodos está dado por la Ecuación 2. 


$$
\text { Num_Est } t_{t}=\beta_{0}+\beta_{1} D_{1 t}+\beta_{2} \text { Num_Cola }+u_{t}
$$

En donde $\beta_{0}$ es el parámetro estimado de intersección del modelo que muestra el valor promedio del número de estrategias que las MIPyMEs llevaron a cabo en el año 2017 cuando la variable dicótoma $D_{1 t}=0$, el parámetro estimado $\beta_{1}$ muestra el efecto diferencial en el promedio del número de estrategias que las MIPyMEs llevaron a cabo en el año 2018 en relación al año 2017 cuando la variable dicótoma $D_{1 t}=1$, de tal forma que $\beta_{0}+\beta_{1}$ es el promedio del número de estrategias que las MIPyMEs llevaron a cabo durante el año 2018 si la variable dicótoma $D_{1 t}=1$.

En el contexto del modelo econométrico ANCOVA la única variable cuantitativa que explica los cambios en el nivel promedio de estrategias competitivas de las MIPyMEs $\left(N u m_{-} E s t_{t}\right)$ es el número de colaboradores $\left(\right.$ Num_Cola $\left._{t}\right)$ la cual puede ser llamada también covariante y su efecto incremental sobre el nivel promedio de estrategias es dado por su parámetro estimado $\beta_{2}$.

El nivel promedio estimado del número de estrategias a partir del número de colaboradores de las MIPyMEs está dado por las Ecuaciones 3 y 4 respectivamente para cada periodo muestral 2017 y 2018.

$$
\begin{aligned}
& \text { Si } D_{1 t}=0 \quad \text { Num_Est }_{t}=\beta_{0}+\beta_{2} \text { Num_Cola }_{t}+u_{t} \\
& \text { Si } D_{1 t}=1 \quad \text { Num_Est } t_{t}=\beta_{0}+\beta_{1} D_{1 t}+\beta_{2} \text { Num_Cola }_{t}+u_{t}
\end{aligned}
$$

Para rechazar la hipótesis nula $H_{0}$ de que no existe relación entre el número de estrategias y el número de colaboradores de las MIPyMEs, es decir $H_{0}: \beta_{2}=0$, se emplea la prueba $t-$ student de significancia individual, cuyo estadístico de prueba se muestra en la Ecuación 5.

$$
t=\beta_{2} / e e\left(\beta_{2}\right)
$$

En donde $\beta_{2}$, es el parámetro estimado del número de colaboradores calculado por mínimos cuadrados ordinarios (MCO) y ee $\left(\beta_{2}\right)$ es su error estándar. Si el valor de $t$ estimado es lo suficientemente grande para ser mayor a su valor $t$ crítico de tablas con un nivel de 
Vásquez Rojas, C.; Martínez Navarrete, D.; Hernández Peralta, A. J.; Villanueva Vásquez, D.

significancia $\alpha$ igual a 5\%, se puede rechazar la hipótesis nula. Una forma práctica de evaluar el rechazo de $H_{0}$ es encontrando el nivel exacto de probabilidad al cual puede rechazarse por medio de su valor de probabilidad $p_{\text {value }}$, si este es menor al nivel de significancia de la prueba preestablecido, entonces se rechaza la hipótesis nula y se acepta que el número de estrategias y el número de colaboradores están relacionados de manera estadísticamente significativa.

\section{Resultados y Discusión}

A lo largo de la aplicación de planes estratégicos en MIPyMEs se ha detectado que el llevar a cabo eficientemente la distribución de todas las estrategias empresariales que surgen en la elaboración del plan estratégico depende del número de colaboradores en una organización.

Se analizaron los diagramas de dispersión de los periodos 2017 y 2018 (Figura 1), posteriormente se realizó una correlación lineal de Pearson entre las variables número de colaboradores y número de estrategias donde se presentó una correlación fuerte y positiva del $71.16 \%$ para la totalidad del periodo, en lo cual se puede observar que la relación entre dichas variables es alta. Cuando se calcula la correlación para cada periodo, la correlación lineal de Pearson es más significativa en el primer año 2017 (78.51\%) que en el segundo año 2018 (48.53\%). La magnitud y el signo de la correlación lineal entre las variables muestran la dependencia positiva que las MIPyMEs presentan del número de colaboradores para incrementar el número de estrategias. 


\section{Figura 1.}

Diagrama de Dispersión Entre las Variables para los dos Periodos Muestrales, 2017 y 2018.
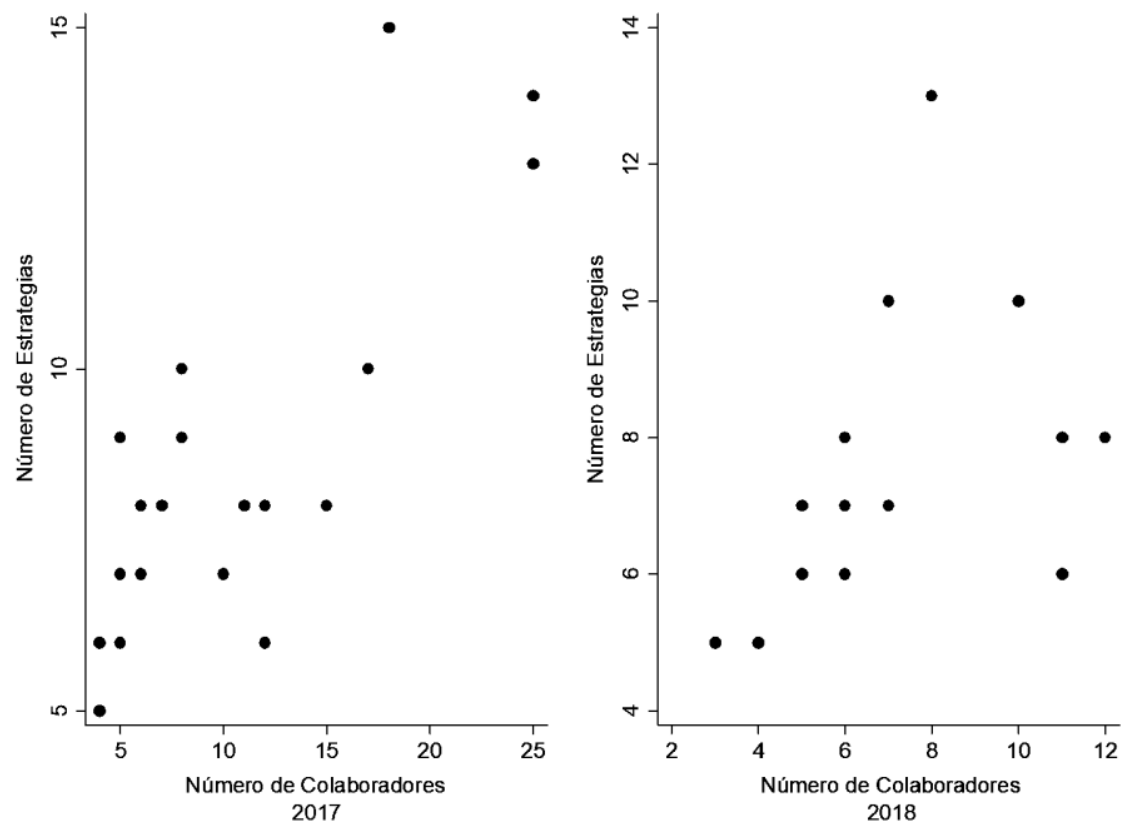

Fuente: Elaboración propia.

Los resultados del modelo econométrico ANCOVA se muestran por medio de la Ecuación 6, se aprecia que el nivel promedio del número de estrategias que implementaron las MIPyMEs durante el 2017 fue de 5 aproximadamente de acuerdo con el valor del parámetro estimado $\beta_{0}$, cuyo valor promedio difiere en una magnitud insignificante al año 2018 con respecto al valor del parámetro estimado $\beta_{1}=0.0116$. Conforme el valor estimado del parámetro $\beta_{2}$ por cada aumento en el número de colaboradores correspondiente a una unidad, manteniendo todo lo demás constante, se estima que en promedio el número de estrategias de las MIPyMEs aumente en 0.3291, este efecto incremental del número de colaboradores en el nivel promedio del número de estrategias que pueden implementar las MIPyMEs es independiente del periodo en el que se observe y al ser positivo confirma la dependencia positiva de las estrategias en función del número de colaboradores (Figura 2).

$$
N u m_{-} E s t_{t}=5.258+0.0116 D_{1 t}+0.3291 N u m_{-} \text {Cola } t+u_{t}
$$


Vásquez Rojas, C.; Martínez Navarrete, D.; Hernández Peralta, A. J.; Villanueva Vásquez, D.

Los resultados de la estimación del modelo por MCO se muestran en la Tabla 3, según el coeficiente de determinación $R^{2}$ casi el $50 \%$ de las variaciones observadas en el número de estrategias son explicadas por las variaciones en el número de colaboradores de las MIPyMEs durante el periodo analizado, lo cual muestra una bondad moderada de ajuste de la información al modelo.

\section{Tabla 3}

Resultados de la Estimación del Modelo Econométrico ANCOVA del Número de Estrategias en Función del Número de Colaboradores

Variable respuesta $\mathbf{N u m} \boldsymbol{E s t}_{\boldsymbol{t}}$ (número de estrategias competitivas llevadas a cabo por las

MYPEs durante 2017 y 2018), nivel de confianza $\boldsymbol{\alpha}=\mathbf{5} \%$ de la prueba $\boldsymbol{t}$ y $\boldsymbol{F}$

\begin{tabular}{|c|c|c|c|c|}
\hline Coeficiente & Error estándar & t-student & Valor $\mathrm{p}$ & $F=19.5$ \\
\hline $\boldsymbol{\beta}_{\mathbf{0}}=5.258679$ & 0.5587113 & 0.02 & 0.983 & Valor $\mathrm{p}=0.000$ \\
\hline $\boldsymbol{\beta}_{1}=0.011679$ & 0.0547671 & 6.01 & 0.000 & \\
\hline $\boldsymbol{\beta}_{2}=0.329168$ & 0.6491967 & 8.10 & 0.000 & $R^{2}=0.4805$ \\
\hline
\end{tabular}

Fuente: Elaboración propia. 


\section{Figura 2.}

Regresión lineal a partir de estimaciones del modelo ANCOVA.

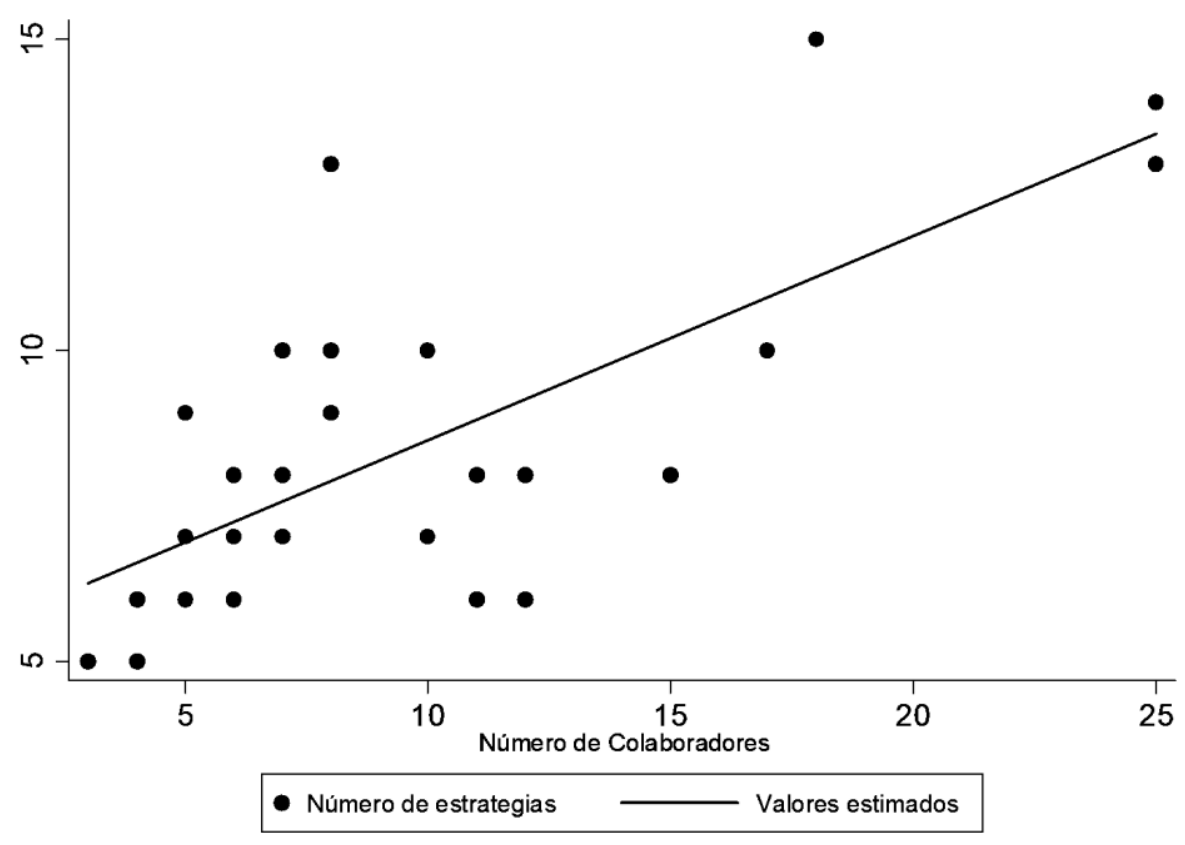

Fuente: Elaboración propia ${ }^{2}$.

La prueba de significancia individual $t$ - student de cada parámetro estimado del modelo, $\beta_{2}$ es estadísticamente significativo en función del $p_{v a l u e}<0.05$ (Tabla 2), por ende se rechaza la hipótesis nula de no relación entre las variables y se confirma que existe una relación y dependencia empírica estadísticamente significativa entre el número de estrategias y el número de colaboradores de las MIPyMEs, para el caso de $\beta_{1}$ este es también estadísticamente significativo con base en su $p_{\text {value }}<0.05$ (Tabla 2) lo cual indica que existen diferencias entre el valor promedio del número de estrategias implementadas por las

\footnotetext{
${ }^{2}$ Modelo de regresión lineal del número de estrategias de las MIPyMEs en función del número de colaboradores a partir de estimaciones del modelo ANCOVA para los periodos 2017 y 2018.
} 
Vásquez Rojas, C.; Martínez Navarrete, D.; Hernández Peralta, A. J.; Villanueva Vásquez, D.

MIPyMEs en cada periodo, esto muestra evidencia de la dinámica de las MIPyMEs para cambiar sus estrategias conforme cambia el número de empleados a través del tiempo (Figura 2). Finalmente, el valor del parámetro estimado $\beta_{0}$ no es estadísticamente significativo lo que indica que el verdadero valor promedio de las estrategias implementadas por las MIPyMEs durante el 2017 fue diferente de aproximandamente 5.

\section{Conclusiones}

Las MIPyMEs han sido objeto de análisis durante las últimas décadas por su contribución en el desarrollo económico de México, sin embargo, son diferentes variables las que inciden en ellas para lograr su permanencia en el mercado. Dentro de estas, el diseño de estrategias considerando los recursos humanos es esencial para mejorar su desempeño (Leal de Valor et al., 2011). Aunque las estrategias tienen un impacto positivo, es importante la incorporación de mejoras en los procesos que sirvan para alcanzar los objetivos empresariales, como se ha mencionado, existe literatura que se enfoca en el desempeño de recursos humanos, pero no ahondan en uno de los obstáculos que enfrentan las MIPyMES que es determinar el número adecuado de estrategias en función del número de colaboradores, y de esta manera puedan reducir la brecha hacia el logro de sus metas.

Una posible solución a la problemática que se origina en la determinación propicia de estrategias empresariales a causa del tamaño de la empresa (número de colaboradores), pueden ser las herramientas que se aplicaron en el presente trabajo; cuando se selecciona un número equivocado de estrategias empresariales el resultado es un ineficiente plan estratégico que, a su vez desencadena una serie de resultados negativos en las metas de la empresa.

La estimación del modelo ANCOVA permitió rechazar la hipótesis nula de que no existe relación entre el número de estrategias empresariales y el número de colaboradores $\left(H_{0}: \beta_{2}=0\right)$ para el caso de las MIPyMES especializadas (actividad económica de restaurantes con servicios de preparación de alimentos a la carta) por medio de la prueba $t$ de significancia individual, dicha relación al ser positiva muestra el estado actual de aprovechamiento y adaptabilidad de los recursos humanos en la determinación de estrategias 
en las MIPyMES para el logro de objetivos. Se halló evidencia estadísticamente significativa a favor de la hipótesis teórica de que el número de estrategias empresariales está relacionado con el número de colaboradores.

El modelo ANCOVA contribuyó a la estimación del número promedio de estrategias empresariales que las MIPyMES pueden establecer dado el número de colaboradores con los que cuenta para llevar a cabo la implementación del plan estratégico; las estrategias no permanecen constantes a través del tiempo, es decir, varían cuando aumenta el número de colaboradores como lo demostró la prueba de significancia individual del parámetro estimado $\beta_{1}$ al ser estadísticamente significativa; aun cuando la diferencia del valor promedio del número de estrategias que llevaron a cabo las empresas durante el año 2018 no fue alto en relación al año 2017. Esto refleja la adaptabilidad y flexibilidad de las MIPyMEs en el entorno global donde interactúan.

Cabe mencionar que no fue posible estimar un número de estrategias óptimo dado el número de colaboradores con los que cuentan las MIPyMEs, sin embargo, auxilia a una mejor toma de decisión que ayuda a la gestión eficiente de sus recursos por medio del modelo de regresión ANCOVA, en consecuencia, el efecto incremental lineal y constante del número de colaboradores en el número de estrategias que el modelo estimó por medio del parámetro $\beta_{2}$, no obstante, ayuda a comprender el grado de respuesta del número de estrategias de las MIPyMEs ante un cambio en el número de colaboradores; considerando el valor estimado $\beta_{2}$, el modelo explica que con tres colaboradores las MIPyMEs pueden añadir una estrategia más a su proceso de planeación, lo cual representa un número relativamente pequeño de insumos que muestra la simplicidad de la estructura de las MIPyMEs para la evaluación de su desempeño a través del cumplimiento de objetivos e indicadores.

Evidentemente, este tipo de análisis podría ser más enriquecedor con un mayor volumen de datos que más adelante pueden ser recopilados, cada vez que se aplique un modelo de planeación estratégica. Este trabajo, permitió establecer un panorama general para aplicar modelos econométricos para la determinación de una mejor decisión del número de estrategias empresariales considerando el número de colaboradores de una empresa. 
Vásquez Rojas, C.; Martínez Navarrete, D.; Hernández Peralta, A. J.; Villanueva Vásquez, D.

\section{Agradecimientos}

Se agradece al Consejo Nacional de Ciencia y Tecnología (Conacyt) y al Programa para el Desarrollo Profesional Docente (PRODEP)

\section{Referencias}

Ahumada Tello, E., \& Perusquia Velasco, J. M. A. (2016). Inteligencia de negocios: Estrategia para el desarrollo de competitividad en empresas de base tecnológica. Contaduria y Administracion. https://doi.org/10.1016/j.cya.2015.09.006

Aït Razouk, A. (2011). High-performance work systems and performance of French smalland medium-sized enterprises: examining causal order. The International Journal of Human Resource Management, 22(2), 311-330. https://doi.org/10.1080/09585192.2011.540157

Amarjit, G., \& Nahum, B. (2012). Barriers to small business growth in Canada. Journal of Small Business and Enterprise Development, 19(4), 656-668. https://doi.org/10.1108/14626001211277451

Antonio, J., Valdez, S., Arodi, O., Laguna, F., Saraí, K., \& Gutiérrez, B. (2020). Factores predictores del modelo de negocio validado por directivos de pequeñas y medianas empresas de monterrey , Nuevo León. 66(2), 1-20. https://doi.org/10.22201/fca.24488410e.2021.2295

Aragón-Sánchez, A., \& Sánchez-Marín, G. (2005). Strategic orientation, management characteristics, and performance: A study of Spanish SMEs. Journal of Small Business Management. https://doi.org/10.1111/j.1540-627X.2005.00138.X

Barney, J. (1991). Firm Resources and Sustained Competitive Advantage. Journal of Management. https://doi.org/10.1177/014920639101700108

Dullayaphut, P., \& Untachai, S. (2013). Development the Measurement of Human Resource Competency in SMEs in Upper Northeastern Region of Thailand. Procedia - Social 
Influencia entre el Número de Colaboradores y Estrategias en las MIPyMEs de la Zona

Centro del Estado de Veracruz

and Behavioral Sciences, $\quad$ 68, https://doi.org/https://doi.org/10.1016/j.sbspro.2013.08.481

Estrada, S., \& Heijs, J. (2018). Comportamiento innovador y competitividad: factores explicativos de la conducta exportadora en México. El caso de Guanajuato. Problemas Del Desarrollo. Revista Latinoamericana de Economía. https://doi.org/10.22201/iiec.20078951e.2005.143.7599

Fiegenbaum, A., \& Karnani, A. (1991). Output flexibility-A competitive advantage for small firms. Strategic Management Journal. https://doi.org/10.1002/smj.4250120203

Gibert, X. (2006). Gestión de recursos humanos. In Gestión Diaria del Hospital (pp. 153165). Elsevier. https://doi.org/10.1016/B978-84-458-1666-0.50009-5

Gujarati, D. N., \& Porter, D. C. (2011). Econometria Básica. In Basic Econometrics. https://doi.org/10.1126/science.1186874

Hitt, M. A., Ireland, R. D., Camp, S. M., \& Sexton, D. L. (2008). Strategic Entrepreneurship: Integrating Entrepreneurial and Strategic Management Perspectives. In Strategic Entrepreneurship: Creating a New https://doi.org/10.1002/9781405164085.ch1

Huitema, B. (2011). The analysis of covariance and alternatives: Statistical methods for experiments, quasi-experiments, and single-case studies ((Vol. 608)).

Ireland, R. D., Hitt, M. A., Camp, S. M., \& Sexton, D. L. (2001). Integrating entrepreneurship and strategic management actions to create firm wealth. Academy of Management Perspectives, 15(1), 49-63. https://doi.org/10.5465/ame.2001.4251393

Islami, X., Mustafa, N., \& Topuzovska Latkovikj, M. (2020). Linking Porter's generic strategies to firm performance. Future Business Journal, 6(1), 3. https://doi.org/10.1186/s43093-020-0009-1

Jaffe, A. B., \& Lerner, J. (2006). Innovation and its discontents. In Policy \& the Economy (MIT Press) (Vol. 6, Issue August). https://doi.org/10.1086/ipe.6.25056179

Jaramillo, S. A. (2019). Planeación estratégica y su aporte al desarrollo empresarial. Espí-ritu Emprendedor TES, 3(1), 64-73. https://doi.org/10.33970/eetes.v3.n1.2019.127 
Vásquez Rojas, C.; Martínez Navarrete, D.; Hernández Peralta, A. J.; Villanueva Vásquez, D.

Knight, G. A. (2001). Entrepreneurship and strategy in the international SME. Journal of International Management. https://doi.org/10.1016/S1075-4253(01)00042-4

Kuratko, D. F., Goodale, J. C., \& Hornsby, J. S. (2001). Quality Practices for a Competitive Advantage in Smaller Firms. Journal of Small Business Management, 39(4), 293311. https://doi.org/10.1111/0447-2778.00027

Leal de Valor, D. Y., Bolívar de Muñoz, M. E., \& Castillo Torrealba, C. O. (2011). La Planificación Estratégica como proceso de integración de un equipo de salud. Enfermería Global, 10(24), 0-0. https://doi.org/10.4321/S1695-61412011000400015

Lohr, S. L. (2015). Coverage and Sampling. In International Handbook of Survey Methodology. https://doi.org/10.4324/9780203843123.ch6

López Fernández, R., Urquiola Sánchez, O., \& Capa Benítez, L. B. (2018). Prácticas de recursos humanos e innovación en las PyMES de alojamiento turístico en Machala, Ecuador: Un estudio exploratorio. PASOS. Revista de Turismo y Patrimonio Cultural, 16(3), 833-842. https://doi.org/10.25145/j.pasos.2018.16.058

Lussier, R. N., \& Sonfield, M. C. (2012). Family businesses’ succession planning: a sevencountry comparison. Journal of Small Business and Enterprise Development, 19(1), 7-19. https://doi.org/10.1108/14626001211196370

Malhotra, N. K. (2012). Basic Marketing Research. In Implicit Measures of Attitudes.

Management, U. S. O. P. (2011). Strategic Human Resources Management: Aligning with the Mission. In HUMAN RESOURCE MANAGEMENT: ISSUES, CHALLENGES AND OPPORTUNITIES.

Messeghem, K. (2003). Strategic Entrepreneurship and Managerial Activities in SMEs. International Small Business Journal. https://doi.org/10.1177/0266242603021002004

Molina Sánchez, R., López Salazar, A., \& Soto Contreras, R. (2014). El emprendimiento y crecimiento de las Pymes. Acta Universitaria. https://doi.org/10.15174/au.2014.701 
Muda, S., \& Rahman, M. R. C. A. (2016). Human Capital in SMEs Life Cycle Perspective. Procedia Economics and Finance, 35, 683-689. https://doi.org/https://doi.org/10.1016/S2212-5671(16)00084-8

Narula, R. (2004). R\&amp;D collaboration by SMEs: new opportunities and limitations in the face of globalisation. Technovation, 24(2), 153-161. https://doi.org/10.1016/S0166-4972(02)00045-7

Obando Changúan, M. P., Cuenca Caraguay2, ; Víctor Emilio, \& Rea Dávalos, M. P. (2020). The organizational culture in Smes and their performance. Prosciences, Vol. 4(32), 47-55. https://doi.org/10.29018/issn.2588-1000vol4iss32.2020pp47-55

Ochoa, C. (2015). Muestreo no probabilístico: muestreo por cuotas. Netquest.

Ong, J. W., Ismail, H. Bin, \& Goh, G. G. G. (2010). The Competitive Advantage of Small and Medium Enterprises (SMEs): The Role of Entrepreneurship and Luck. Journal of Small Business \& Entrepreneurship, 23(3), 373-391. https://doi.org/10.1080/08276331.2010.10593491

Otzen, T., \& Manterola, C. (2017). Técnicas de Muestreo sobre una Población a Estudio. International Journal of Morphology. https://doi.org/10.4067/S0717$\underline{95022017000100037}$

Palombino Primo, P., Oliva, E. de C., \& Kubo, E. K. de M. (2014). Gestão estratégica de pessoas para pesquisadores em administração nas universidades privadas. REAd. Revista Eletrônica de Administração (Porto Alegre), 20(2), 371-396. https://doi.org/10.1590/1413-2311063201238442

Patel, P. C., \& Cardon, M. S. (2010). Adopting HRM practices and their effectiveness in small firms facing product-market competition. Human Resource Management, 49(2), 265-290. https://doi.org/https://doi.org/10.1002/hrm.20346

Pérez-Elizundia, G., Delgado-Guzmán, J. A., \& Lampón, J. F. (2020). Commercial banking as a key factor for SMEs development in Mexico through factoring: A qualitative approach. European Research on Management and Business Economics, 26(3), 155163. https://doi.org/https://doi.org/10.1016/j.iedeen.2020.06.001 
Vásquez Rojas, C.; Martínez Navarrete, D.; Hernández Peralta, A. J.; Villanueva Vásquez, D.

Pil, F. K., \& Holweg, M. (2003). Exploring Scale: The Advantages of Thinking Small. MIT Sloan Management Review. 44(2):33-39

Rheinheimer, D. C., \& Penfield, D. A. (2001). The Effects of Type I Error Rate and Power of the ANCOVA F Test and Selected Alternatives Under Nonnormality and Variance Heterogeneity. The Journal of Experimental Education, 69(4), 373-391. https://doi.org/10.1080/00220970109599493

Rocca, García, \& Gómez. (2016). Factores determinantes del éxito competitivo en la mipyme: un estudio empírico en empresas peruanas. Contabilidad y Negocios. 11(22), 52-68. https://doi.org/10.18800/contabilidad.201602.004

Santini, S. M. L., Nunes, E. de F. P. de A., Carvalho, B. G., \& Souza, F. E. A. de. (2017). DOS 'RECURSOS HUMANOS' À GESTÃO DO TRABALHO: UMA ANÁLISE DA LITERATURA SOBRE O TRABALHO NO SUS. Trabalho, Educação e Saúde, 15(2), 537-559. https://doi.org/10.1590/1981-7746-sol00065

Sheehan, M. (2013). Human resource management and performance: Evidence from small and medium-sized firms. International Small Business Journal, 32(5), 545-570. https://doi.org/10.1177/0266242612465454

Shieh, G. (2020). Power Analysis and Sample Size Planning in ANCOVA Designs. Psychometrika, 85(1), 101-120. https://doi.org/10.1007/s11336-019-09692-3

Valente, M. J., \& MacKinnon, D. P. (2017). Comparing Models of Change to Estimate the Mediated Effect in the Pretest-Posttest Control Group Design. Structural Equation Modeling: A Multidisciplinary Journal, 24(3), 428-450. https://doi.org/10.1080/10705511.2016.1274657

Van Breukelen, G. J. P. (2013). ANCOVA Versus CHANGE From Baseline in Nonrandomized Studies: The Difference. Multivariate Behavioral Research, 48(6), 895-922. https://doi.org/10.1080/00273171.2013.831743

Vrgovic, P., Vidicki, P., Glassman, B., \& Walton, A. (2012). Open innovation for SMEs in developing countries - An intermediated communication network model for 
Influencia entre el Número de Colaboradores y Estrategias en las MIPyMEs de la Zona Centro del Estado de Veracruz

collaboration beyond obstacles. Innovation: Management, Policy and Practice, 14(3), 290-302. https://doi.org/10.5172/impp.2012.14.3.290

Wang, C. K., \& Ang, B. L. (2004). Determinants of venture performance in Singapore. In Journal of Small Business Management. 42(4), 347-363 https://doi.org/10.1111/j.1540-627X.2004.00116.X

Williams, R. J., Schnake, M. E., \& Fredenberger, W. (2005). The Impact of Corporate Strategy on a Firm's Reputation. Corporate Reputation Review, 8(3), 187-197. https://doi.org/10.1057/palgrave.crr.1540249

Zaridis, A. D., \& Mousiolis, D. T. (2014). Entrepreneurship and SME's Organizational Structure. Elements of a Successful Business. Procedia - Social and Behavioral, 148, 463-467. https://doi.org/10.1016/j.sbspro.2014.07.066 